\title{
Density of States Measured by Scanning-Tunneling Spectroscopy Sheds New Light on the Optical Transitions in PbSe Nanocrystals
}

\author{
Peter Liljeroth, ${ }^{1, *}$ Pedro A. Zeijlmans van Emmichoven, ${ }^{1}$ Stephen G. Hickey, ${ }^{2}$ Horst Weller, ${ }^{2}$ Bruno Grandidier, ${ }^{3}$ \\ Guy Allan, ${ }^{3}$ and Daniël Vanmaekelbergh ${ }^{1}$ \\ ${ }^{1}$ Debye Institute, University of Utrecht, PO Box 80000, 3508 TA Utrecht, The Netherlands \\ ${ }^{2}$ Institute of Physical Chemistry, University of Hamburg, Grindelallee 117, D-20146 Hamburg, Germany \\ ${ }^{3}$ IEMN (CNRS, UMR 8520), Département ISEN, 41 bd Vauban, 59046 Lille Cedex, France
}

(Received 15 March 2005; published 17 August 2005)

\begin{abstract}
The density-of-states function of individual colloidal PbSe nanocrystals varying in diameter between 3 and $7 \mathrm{~nm}$ is measured by resonant tunneling spectroscopy. It is in semiquantitative agreement with tightbinding calculations, but the energy separation between electron (hole) levels of $S$ and $P$ symmetry is systematically smaller than predicted by the theory. These results provide an explanation for the second and third excitonic optical transitions, which have been debated for a long time.
\end{abstract}

DOI: 10.1103/PhysRevLett.95.086801

PACS numbers: 73.22. $-\mathrm{f}, 73.63 .-\mathrm{b}, 78.67 .-\mathrm{n}$

The lead chalcogenides ( $\mathrm{PbS}, \mathrm{PbSe}$, and $\mathrm{PbTe}$ ) have a rock salt crystal structure; as a result, the fundamental band gap is located at the four $L$ points in the Brillouin zone. This has noticeable consequences for nanocrystals (NCs), in which the charge carrier wave functions are strongly confined: the highest valence (hole) level (HOMO) and the lowest conduction (electron) level (LUMO) become eightfold degenerate [1-4]. The other discrete energy levels also have a fourfold higher multiplicity with respect to the corresponding energy levels of II-VI and III-V quantum dots (QDs).

Related to their electronic structure and high dielectric constant, lead chalcogenide NCs possess a number of optical properties of high potential for future optoelectronic applications, such as LEDs, lasers, and solar cells [1,5-9]. Despite this, the electronic structure of the lead chalcogenides in the regime of strong confinement has received relatively little attention. In an early paper, Kang and Wise reported on the discrete energy levels of $\mathrm{PbS}$ and $\mathrm{PbSe}$ QDs calculated by an effective mass approximation [4]. The calculated spectrum shows three discrete (but multiply degenerate) conduction energy levels, the envelope wave functions having $S, P$, and $D$ symmetries, respectively (denoted as $S_{\mathrm{e}}, P_{\mathrm{e}}$, and $D_{\mathrm{e}}$ ). The structure of the valence levels mirrors that of the conduction levels, i.e., $S_{\mathrm{h}}, P_{\mathrm{h}}$, and $D_{\mathrm{h}}$. The energy levels of realistic, faceted $\mathrm{PbSe}$ NCs have only very recently been calculated with a stateof-the-art tight-binding (TB) model [3]. This calculation shows a group of eight $S$-type conduction levels, followed by $24 P$-type levels, and a quasicontinuum of closely spaced levels at higher energy. The energy spread within a group increases (up to $50 \mathrm{meV}$ with $S$ levels) when the diameter of the NC decreases from 10 to $2 \mathrm{~nm}$. The valence hole levels consist of a group of eight $S$-type levels; however, the group of $P$ levels is split into two.

These detailed calculations highlighted unexplained features in the optical absorption spectrum of colloidal $\mathrm{PbSe}$
NCs. The second exciton resonance is at odds with theoretical predictions, in energy, it corresponds to $S_{\mathrm{h}}-P_{\mathrm{e}}$ or $S_{\mathrm{e}}-P_{\mathrm{h}}$ separations calculated by TB; however, such transitions are parity forbidden [3]. The nature of this transition has been discussed extensively in the literature [2$4,6,8,10]$.

In this Letter, we report the single-particle density-ofstates function of colloidal PbSe NCs obtained with resonant tunneling spectroscopy using an STM at $5 \mathrm{~K}$. This technique has been previously used to measure the singleparticle energy levels and electron-electron interactions in colloidal and strain-induced InAs [11-14] and colloidal CdSe NCs [15] as well as electrochemically grown $\mathrm{PbS}$ and $\mathrm{PbSe}$ QDs $[16,17]$. It has been shown that interparticle Coulomb repulsion can be avoided under conditions of shell-tunneling; i.e., tunneling into the QD is much slower than tunneling out of the dot $[14,15,18]$. We measure the single-particle density-of-states function around the fundamental gap for a large number of QDs of diameters between 3 and $7 \mathrm{~nm}$. The spectral features are in semiquantitative agreement with the results of TB calculations [3]. We find, however, that the energy separation between the LUMO $S_{\mathrm{e}}$ and $P_{\mathrm{e}}$ group, and the HOMO $S_{\mathrm{h}}$ and $P_{\mathrm{h}}$ group, are systematically smaller than predicted by TB calculations. Our results indicate that the second resonance in the absorption spectrum corresponds to the allowed $P_{\mathrm{e}}-P_{\mathrm{h}}$ transition instead of the forbidden $S_{\mathrm{e}}-P_{\mathrm{h}}$ or $P_{\mathrm{e}}-S_{\mathrm{h}}$ transitions. The measured density-of-states function could thus provide a solution for a long-standing controversy concerning the optical transitions in PbSe QDs [2-4,6,8,10].

A flame-annealed $\mathrm{Au}(111)$ substrate with a selfassembled hexanedithiol monolayer [19] was immersed in a dispersion of colloidal PbSe NCs (synthesized according to Ref. [9] and recapped using hexylamine) for $1 \mathrm{~min}$. This lead to stable attachment of individual PbSe NCs and the formation of small aggregates of a few PbSe QDs [Fig. 1(a)]. Two different samples with PbSe NCs of aver- 

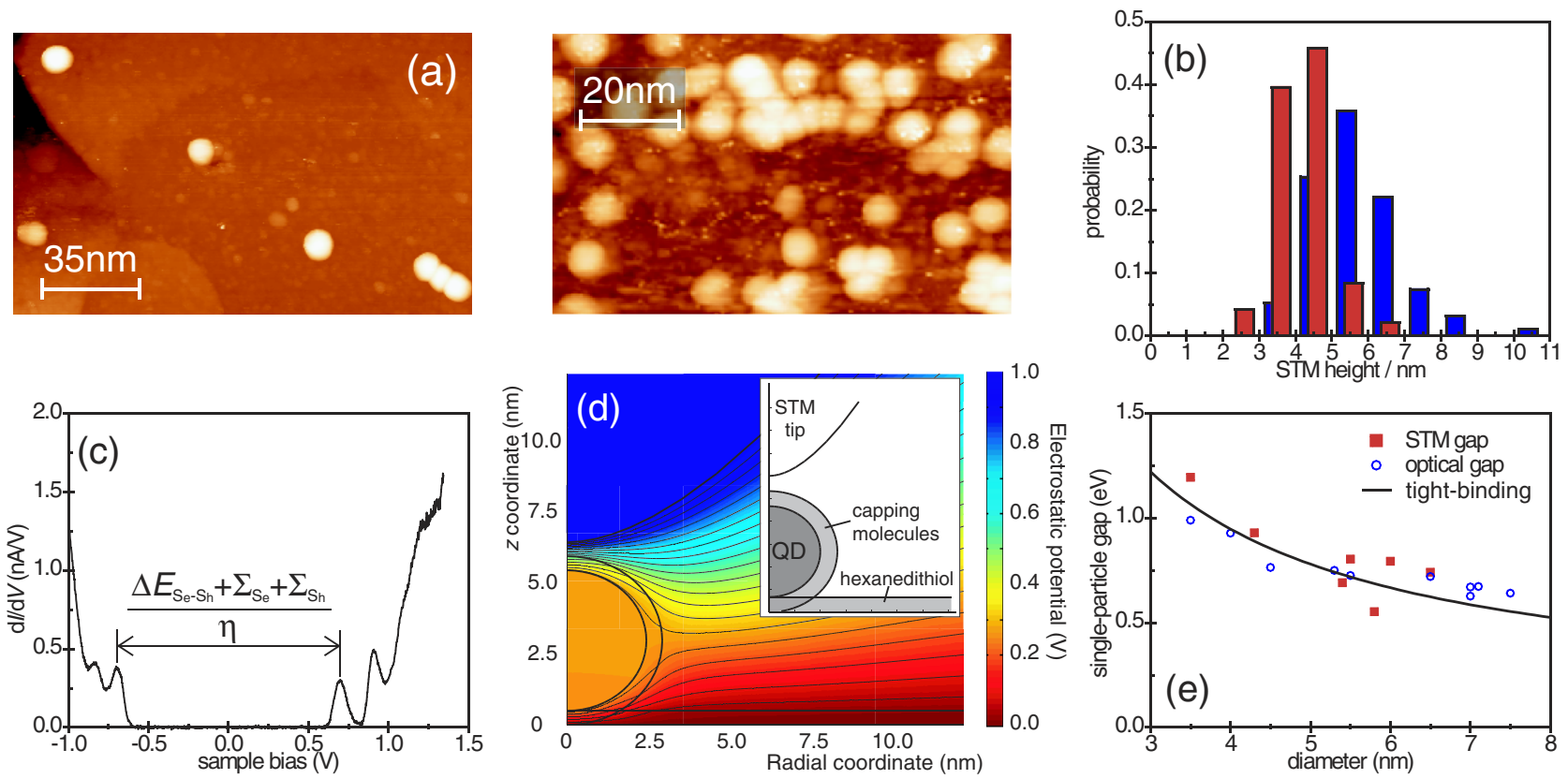

FIG. 1 (color online). Large scale scans of (a) $5.3 \mathrm{~nm}$ (left) and $4 \mathrm{~nm}$ (right) PbSe NCs covalently bound to a Au substrate. STM parameters $V_{\text {bias }}=1.5 \mathrm{~V}, I_{\text {set-point }}=10 \mathrm{pA}(5.3 \mathrm{~nm} \mathrm{NCs})$, and $2.5 \mathrm{~V} / 10 \mathrm{pA}(4.0 \mathrm{~nm})$. (b) Histogram of particle heights extracted from STM topography for the two samples with TEM determined core sizes of 4.0 and $5.3 \mathrm{~nm}$. (c) Example of a measured tunneling spectrum $(d I / d V, V)$ on an isolated PbSe NC. (d) Potential distribution in the tip/QD/substrate junction calculated by the solution of the Poisson equation. (e) Comparison of the single-particle HOMO-LUMO gap measured by STM (filled squares, $\eta=0.7, \epsilon_{\mathrm{out}}^{\text {eff }}=3$ ), the optical gap (open circles, our data and data from Refs. [8,9]), and the single-particle gap from TB calculations [3] (solid line).

age diameters of 4.0 and $5.3 \mathrm{~nm}$ (measured by TEM) were investigated. Histograms of the height of the NCs measured from the topographic STM images are shown in Fig. 1(b). It is clear that the average height of the $\mathrm{PbSe}$ QDs is in fair agreement with the core diameter from the TEM analysis. This implies that we can use the apparent STM height of an individual $\mathrm{PbSe}$ QD as an indication of its size. From the histograms, it is also clear that individual $\mathrm{PbSe}$ NCs can be found with a considerable deviation from the average size. In this way, we could select individual NCs of different sizes between 3 and $7 \mathrm{~nm}$, for resonant tunneling spectroscopy.

Tunneling spectra were acquired at $5 \mathrm{~K}$ in an ultrahigh vacuum STM (Omicron LT STM, base pressure $\sim 10^{-10}$ mbar) by placing the tip above the center of an individual $\mathrm{PbSe} \mathrm{QD}$ and disconnecting the feedback loop. The tunneling current, $I$, and conductance, $d I / d V$, were acquired simultaneously (rms modulation $6 \mathrm{mV}$ at $1 \mathrm{kHz}$ ). Typically, a large number (20-100) of $(I, V)$ and $(d I / d V$, $V)$ traces were acquired above a single dot, and checked for their reproducibility. In general, the conductance spectra were very reproducible, although discrete shifts of the spectra along the voltage axis were observed in some cases. Sets of reproducible $(I, V)$ and $(d I / d V, V)$ plots were averaged to increase the signal to noise ratio. This did not affect the width of the resonance peaks. Figure 1(c) shows a typical conductance spectrum acquired with a $\mathrm{PbSe}$ QD with an STM height of $5.5 \mathrm{~nm}$. The spectrum shows several relatively broad tunneling resonances on both sides of the zero-conductance gap. The spectrum remained unaffected on changing the tip-to-dot distance by the set-point current (bias $\sim 1.0-1.5 \mathrm{~V}$ and current $\sim 100-200 \mathrm{pA}$ ). Furthermore, the spectra are in strong contrast to those acquired with PbSe QDs on a HOPG substrate, where multiple (5 to 10 ), equally spaced tunneling resonances reflect single-electron charging of the $\mathrm{PbSe}$ QDs (shell-filling conditions, unpublished data). Thus, we conclude that the conductance spectrum presented in Fig. 1(c) is obtained under shell-tunneling conditions and directly reflects the single-particle density-of-states of the $\mathrm{PbSe}$ NC.

In order to obtain quantitative information from the tunneling spectra, the distribution of the bias voltage over the double-barrier tunnel junction has to be known. We calculate this by solving the Poisson equation for a realistic tip-dot-substrate geometry as shown in Fig. 1(d) [dielectric constants $227(\mathrm{PbSe})$ and 2 (for organic molecules)]. We found that $\eta=\left(V_{\text {dot }}-V_{\text {tip }}\right) / V_{\text {bias }}$ is close to 0.7 for dot sizes between 3 and $7 \mathrm{~nm}$ and realistic tip-to-dot and dotto-substrate distances (tip-dot 5-15 $\AA$ and dot-substrate $5 \AA$ ).

The bias voltage at the resonances in the tunneling spectrum is related to the energy levels $\left(E_{i}\right)$ through $\eta V_{\text {bias }}=E_{i}+\Sigma_{i}$, where $\Sigma_{i}$ is the polarization (charging) energy of an electron on level $i$. This energy depends on the dielectric mismatch between the NC core and its surround- 
ings and can be estimated given the effective dielectric constants [18,20-22]. For NCs used in this study, the polarization energy varies between $70 \mathrm{meV}$ (diameter $7 \mathrm{~nm}$ ) and $160 \mathrm{meV}$ (diameter $3 \mathrm{~nm}$ ) for $\epsilon_{\text {in }}=227$ and $\epsilon_{\text {out }}^{\text {eff }}=3$. The zero-conductance gap in the tunneling spectrum, $\Delta V_{\mathrm{zc}}^{\mathrm{STM}}$, is then related to the single-particle HOMOLUMO gap, $\Delta E_{S_{\mathrm{e}}-S_{\mathrm{h}}}$, as follows: $\eta \Delta V_{\mathrm{Zc}}^{\mathrm{STM}}=\Delta E_{S_{\mathrm{e}}-S_{\mathrm{h}}}+$ $\Sigma_{S_{\mathrm{e}}}+\Sigma_{S_{\mathrm{h}}}$. In Fig. 1(e), the $S_{\mathrm{e}}-S_{\mathrm{h}}$ level separation is shown as a function of the NC height measured by STM. Furthermore, the $S_{\mathrm{h}}-S_{\mathrm{e}}$ band gaps are compared with the optical gaps as a function of the NC diameter derived from TEM analysis. For NCs with a high dielectric constant, the optical gap is expected to be nearly equal to the singleparticle gap $[15,21,22]$. There is a good agreement between the single-particle gap derived from tunneling spectroscopy, the optical gaps, and the single-particle gap calculated from TB theory. This indicates that the STM height is a reliable measure for the actual $\mathrm{NC}$ diameter and that the calculated potential distribution across the tip/dot/substrate junction is realistic.

In Fig. 2, we show $d \ln I / d \ln V$ (proportional to the density of states [17]) for two PbSe NCs with STM heights of 5.5 and $4.3 \mathrm{~nm}$. The bias voltage has been converted to the energy scale using $\eta=0.7$, and polarization energies were calculated with $\epsilon_{\text {out }}^{\text {eff }}=3$; see above. Figure 2 also shows the energy levels calculated by TB (gray lines), obtained by broadening each level (thin solid lines) by a $25 \mathrm{meV}$ Gaussian. The first two resonances on each side of the gap are assigned to the $S_{\mathrm{e}}\left(S_{\mathrm{h}}\right)$ and $P_{\mathrm{e}}\left(P_{\mathrm{h}}\right)$ levels, respectively. The resonances at higher energy are often considerably broader; they correspond to the group of $D$ levels [23]. The agreement of the energy level positions is good on a semiquantitative level; however, the level separations are overestimated by TB. We find this deviation to be systematic by inspecting a larger set of data (see below).

In the following, we will make a detailed comparison between the optical transition energies and the energy level

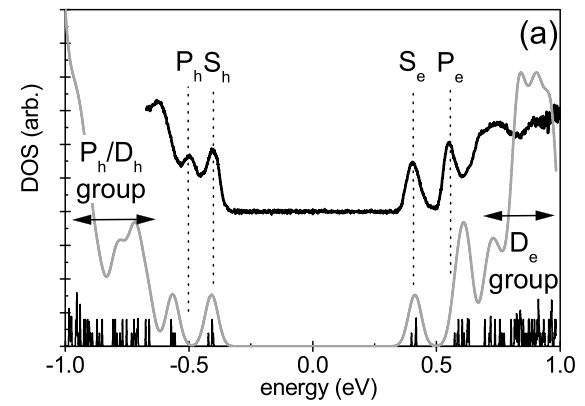

positions measured by tunneling spectroscopy. It must be realized that calculations and optical experiments are performed on isolated nanocrystals. Tunneling experiments are carried out in a double barrier junction geometry. The presence of a self-assembled hexanedithiol monolayer on the gold substrate and the QD capping layer ensures that the QD is sufficiently decoupled from the substrate to allow a comparison between tunneling experiments, optical measurements, and TB. The optical absorbance spectra of high-quality $\mathrm{PbSe} \mathrm{NCs}$ show three excitonic transitions [see inset of Fig. 3(a)]; in the literature, these have been attributed to $S_{\mathrm{e}}-S_{\mathrm{h}}$ (peak 1), $S_{\mathrm{e}}-P_{\mathrm{h}}$ and $P_{\mathrm{e}}-S_{\mathrm{h}}$ (peak 2), and $P_{\mathrm{e}}-P_{\mathrm{h}}$ (peak 3) transitions $[4,6,8,10]$. In Fig. 3(a), the energies of the second and third optical transitions have been plotted as a function of the first exciton energy. The points are based on our measurements and literature data $[8,9]$. These are compared with energy levels derived from the resonant tunneling spectra measurements: the $P_{\mathrm{e}}-P_{\mathrm{h}}$ separations have been plotted as a function of $S_{\mathrm{e}}-S_{\mathrm{h}}$ separation. It is evident that the second excitonic transition corresponds to the $P_{\mathrm{e}}-P_{\mathrm{h}}$ energy level separation measured by STM. This assignment is consistent with the expected selection rules for interband transitions in $\mathrm{PbSe} \mathrm{NCs}$ $[3,10]$. In Fig. 3, we also include the energy separation between the third and the fourth resonance on the positive and negative bias, respectively, and compare it to the third optical resonance. The agreement in energy is remarkable, suggesting that the third optical resonance involves levels of the $D_{\mathrm{e}^{-}}$and $D_{\mathrm{h}}$ groups [24].

Another way of presenting the optical data has been used by Guyot-Sionnest and co-workers [8]; they plot the confinement energy of a given transition $i$ - $j$ scaled by the confinement energy of the first transition as a function of the confinement energy of the first transition: $\left(\Delta E_{i-j}-E_{\text {gap }}\right) /$ $\left(\Delta E_{S_{\mathrm{e}}-S_{\mathrm{h}}}-E_{\text {gap }}\right)$ versus $\Delta E_{S_{\mathrm{e}}-S_{\mathrm{h}}}-E_{\text {gap }}$, where $E_{\text {gap }}$ is the bulk band gap of $\mathrm{PbSe}$ at a given temperature. For the optical transitions, these scaled transition energies have been shown to be approximately independent of the size

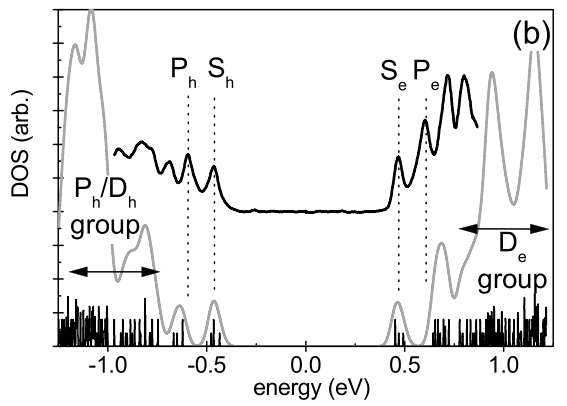

FIG. 2. Comparison between measured $d \ln I / d \ln V$ (solid line) and the density of states calculated by TB [gray line, obtained by broadening each level (thin solid lines) by $25 \mathrm{meV}$ Gaussian] for different NC sizes: (a) STM height $5.5 \mathrm{~nm}$ and (b) $4.3 \mathrm{~nm}$. The TB calculations have been performed for dots of diameters of (a) $4.87 \mathrm{~nm}$ and (b) $4.26 \mathrm{~nm}$; the STM spectra were converted to an energy scale using $\eta=0.7$ and $\epsilon_{\mathrm{out}}^{\text {eff }}=3$. The assignment of the first two resonances $\left(S_{\mathrm{e}}, P_{\mathrm{e}}, S_{\mathrm{h}}\right.$, and $\left.P_{\mathrm{h}}\right)$ and the groups of $D$ levels are indicated. 

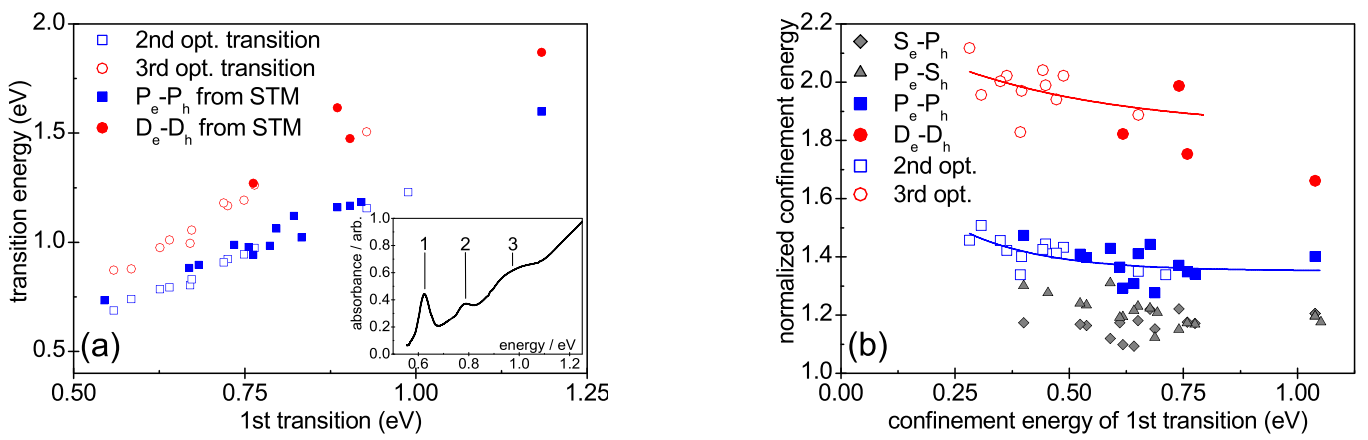

FIG. 3 (color online). Comparison between the observed optical transitions and the energy levels measured by resonant tunneling spectroscopy. (a) The transition energies have been plotted as a function of the energy of the first transition. (b) Normalized confinement energy of a transition $i-j,\left(\Delta E_{i-j}-E_{\mathrm{gap}}\right) /\left(\Delta E_{S_{\mathrm{e}}-S_{\mathrm{h}}}-E_{\mathrm{gap}}\right)$ as a function of the confinement energy of the first transition, $\Delta E_{S_{\mathrm{e}}-S_{\mathrm{h}}}-E_{\text {gap }}$. Filled symbols from STM measurements, open symbols from optical data (our measurements and data from Refs. $[8,9])$. Lines are drawn as a guide to the eye.

of the QDs and of temperature [8]. Figure 3(b) shows the comparison between optical absorbance and STM experiments. The $S_{\mathrm{e}}-P_{\mathrm{h}}$ and $P_{\mathrm{e}}-S_{\mathrm{h}}$ separations derived from the tunneling spectra are significantly below the optical transition energy. This forms a strong indication that the second exciton resonance does not correspond to an $S$ to $P$ transition. We have carefully checked whether TB calculations predict a relaxation of the selection rules for $\mathrm{PbSe}$ nanocrystals lacking spherical or cubic symmetry. Although the forbidden $S_{\mathrm{e}}-P_{\mathrm{h}}$ and $P_{\mathrm{e}}-S_{\mathrm{h}}$ transitions gain some oscillator strength, they are still orders of magnitude weaker than the allowed $S_{\mathrm{e}}-S_{\mathrm{h}}$ and $P_{\mathrm{e}}-P_{\mathrm{h}}$ transitions. A lowering of the nanocrystal symmetry can thus not explain the observed optical transitions.

In summary, we have measured the density-of-states function of colloidal $\mathrm{PbSe} \mathrm{NCs}$ of diameters between 3 and $7 \mathrm{~nm}$ with resonant shell-tunneling spectroscopy. The features of the density-of-states function, i.e., band gap, groups of $S_{\mathrm{e}}, S_{\mathrm{h}}, P_{\mathrm{e}}$, and $P_{\mathrm{h}}$ levels agree semiquantitatively with TB calculations. These data have been used to reappraise the optical absorbance spectra of PbSe NCs: the second transition in the optical absorbance spectrum can be assigned to allowed transition between the group of conduction and valence levels with $P$ symmetry. This work highlights the possibilities offered by STM in measuring the complete density-of-states function, in the absence of selection rules, of individual QDs and relating it to the simultaneously measured geometry.

The authors gratefully acknowledge financial support from the European Union (through EU network "NANOSPECTRA," HPRH-CT-2001-00320).

*Electronic address: P.Liljeroth@ phys.uu.nl [1] F. W. Wise, Acc. Chem. Res. 33, 773 (2000).
[2] A.D. Andreev and A. A. Lipovskii, Phys. Rev. B 59, 15402 (1999).

[3] G. Allan and C. Delerue, Phys. Rev. B 70, 245321 (2004).

[4] I. Kang and F. W. Wise, J. Opt. Soc. Am. B 14, 1632 (1997).

[5] R. D. Schaller and V.I. Klimov, Phys. Rev. Lett. 92, 186601 (2004).

[6] B. L. Wehrenberg and P. Guyot-Sionnest, J. Am. Chem. Soc. 125, 7806 (2003).

[7] Z. Hens et al., Phys. Rev. Lett. 92, 026808 (2004).

[8] B. L. Wehrenberg, C. Wang, and P. Guyot-Sionnest, J. Phys. Chem. B 106, 10634 (2002).

[9] C. B. Murray et al., IBM J. Res. Dev. 45, 47 (2001).

[10] H. Du et al., Nano Lett. 2, 1321 (2002).

[11] U. Banin et al., Nature (London) 400, 542 (1999).

[12] O. Millo et al., Phys. Rev. B 61, 16773 (2000).

[13] O. Millo et al., Phys. Rev. Lett. 86, 5751 (2001).

[14] T. Maltezopoulos et al., Phys. Rev. Lett. 91, 196804 (2003).

[15] E. P. A. M. Bakkers et al., Nano Lett. 1, 551 (2001).

[16] Z. Hens et al., Phys. Rev. Lett. 88, 236803 (2002).

[17] Z. Hens et al., Europhys. Lett. 65, 809 (2004).

[18] Y. M. Niquet et al., Phys. Rev. B 65, 165334 (2002).

[19] V. L. Colvin, A. N. Goldstein, and A. P. Alivisatos, J. Am. Chem. Soc. 114, 5221 (1992).

[20] M. Lannoo, C. Delerue, and G. Allan, Phys. Rev. Lett. 74, 3415 (1995).

[21] A. Franceschetti, A. Williamson, and A. Zunger, J. Phys. Chem. B 104, 3398 (2000).

[22] C. Delerue and M. Lannoo, Nanostructures Theory and Modelling (Springer-Verlag, Berlin, 2004).

[23] According to the TB calculations, the hole $P$ levels are split into two groups. Here we refer to the first of these two groups.

[24] Care should be taken here as the $D$ levels are spread over a considerable energy range and all of these levels are not expected to contribute equally to the tunneling current or to the optical transitions. 